\title{
Two-Dimensional Ising Model and Local Nonuniversality of Critical Exponents
}

\author{
R. Z. Bariev ${ }^{1,2}$ \\ ${ }^{1}$ Departamento de Física, Universidade Federal de São Carlos, 13565-905, São Carlos, SP Brazil \\ ${ }^{2}$ The Kazan Physico-Technical Institute of the Russian Academy of Sciences, Kazan 420029, Russia
}

Received on 15 August, 2000

\begin{abstract}
We obtain the local magnetization of a planar Ising model with defects of different types. It is shown that near the critical point the local magnetization has a nonuniversal behavior that manifests itself in the fact that its critical exponent is a continuous function of the microscopic parameters of the system.
\end{abstract}

The Ising model is the simplest model of magnetism and was proposed by Ising [1] in 1925. The most interesting property of this model was discovered by Onsager [2] for the two-dimensional model in 1944. This model has a phase transition and was the basis for the modern theory of phase transitions. The 8-vertex model was solved by Baxter [3] and has a nonuniversal behavior that manifests itself in the fact that its critical index $\alpha$ is a continuous function of the microscopic parameters of the system. In this report we present a rigorous and phenomenological analysis of different types of local magnetization of the two-dimensional Ising model. Firstly we consider linear defects of two different types [4]. The local magnetization is calculated exactly for this model, and a study is made of its dependence on the distance to the defect and on the size of the defect. The most interesting feature of the solution is the dependence of the critical exponent of the local magnetization on the interaction parameter.

The interaction energy of the lattices considered can be represented as a sum of two terms

$$
E=E_{0}+\Delta E
$$

where $E_{0}$ is the energy of the defect-free lattice,

$E_{0}=-\sum_{m=1-M}^{M} \sum_{n=1-N}^{N}\left(J_{1} s_{m n} s_{m, n+1}+J_{2} s_{m n} s_{m+1, n}\right)$,

and $\Delta E$ is the energy of the perturbation due to the presence of the defects. For the lattice of the first type,

$$
\Delta E=-\left(J_{1}^{\prime}-J_{1}\right) \sum_{m=1-M}^{M} s_{m o} s_{M 1},
$$

and for the lattice of the second type,

$$
\Delta E=-\left(J_{2}^{\prime}-J_{2}\right) \sum_{n=1-n}^{M} s_{m o} s_{N 1} \text {. }
$$

In these expressions $s_{m n}$ is the spin variable connected with a lattice site having coordinates $m$ and $n$, and takes values $\pm 1 ; J_{1}, J_{1}^{\prime}$ and $J_{2}, J_{2}^{\prime}$ are the energies of the interaction between the horizontal and vertical pairs of neighboring spins, respectively. Here both directions $M, N \rightarrow \infty$.

We define the local magnetization of a lattice with defects in analogy with the definition of the magnetization of the spin-spin correlation function

$$
<s_{n}>=\lim _{L \rightarrow \infty}<s_{m n} s_{m, n+L}>/<s_{m, n+L}>.
$$

We have ommitted from the left-hand side of this expression the row index because of the translational invariance of the lattice in question in the vertical direction.

For spins with large column number the local magnetization does not differ from the magnetization < $s>_{0}$ of a defect-free lattice

$$
\begin{aligned}
<s_{0}> & =\left[1-\left(\sinh 2 K_{1} \sinh 2 K_{2}\right)^{-2}\right] \sim \tau^{1 / 8} \\
\tau & =\left|1-T / T_{c}\right|
\end{aligned}
$$

$K_{i}=J_{i} / k T$, where $k$ is Boltzmann's constant, $\mathrm{T}$ is the temperature, and the subscript $c$ marks the value of the function at the critical point $T_{c}$. We have obtained an exact solution for the local magnetization and consider its behavior in the most interesting - scaling - region, defined by the following relations:

$$
\xi^{-1} \rightarrow 0, n \rightarrow \infty
$$

where $\xi$ is the correlation radius of the defect-free lattice

$$
\xi^{-1} \sim \tau
$$


An analysis of the exact expression shows that the local magnetization in this region has the scaling form

$$
<s_{n}>=<s>_{0} f(x, \kappa)
$$

For the lattice with defects of the first type

$$
\begin{aligned}
x & =(2 n-1) \xi^{-1} S_{1}^{-1 / 2}, \\
\kappa & =\left(C_{1}-C_{1}^{\prime}\right)\left(C_{1} C_{1}^{\prime}\right)^{-1},-C_{1}^{-1} \leq \kappa<1 \\
x & =2(n-1) \xi^{-1} S_{1}^{-1 / 2} \\
\kappa & =\tanh 2\left(K_{2}-K_{2}^{\prime}\right),-1<\kappa<1, \\
C_{i} & =\cosh 2 K_{i}, S_{i}=\sinh 2 K_{i} .
\end{aligned}
$$

The most interesting feature of this solution is the nonuniversal behavior of the local magnetization at distances $n$ smaller than half the correlation radius $\xi$ of the defect-free model. This nonuniversality consists in the fact that the critical exponent $\beta_{l o c}$ of the magnetization is a continuous function of the interaction parameters

$$
<s_{n}>\sim \tau^{\beta_{l o c}} \text { for } n \ll \xi,
$$

where

$$
\beta_{l o c}=[\arccos (-\kappa)]^{2} .
$$

Thus, the local magnetization of a lattice with a line of defects, at distances larger than $\xi$ to the defect, hardly differs from the magnetization of a defect-free lattice. The deviation becomes so substantial that it leads to a change of the critical exponent $\beta_{l o c}$ that characterizes the dependence of the local magnetization on the relative temperature $\tau$.

We now discuss the reason for the nonuniversality of the critical behavior of the local magnetization from the point of view of the general theory of phase transitions [5]. Let us investigate a d-dimensional system near a critical point determined by the Hamiltonian

$$
H=H_{0}+\lambda H_{1}=\int \epsilon(r) d r+\int \lambda \omega(r) d r
$$

with $\lambda$ a small parameter. The energy density $\epsilon(r)$ and perturbation density $\omega(r)$ are local quantities that depend on the microscopic variables which describe the system.

We investigate a d-dimensional system near a critical point determined by the Hamiltonian (1). We assume that the perturbation is caused by the presence of a homogeneous defect of dimensionality $d^{*}$. In this case the perturbation itself has dimensionality $d^{\prime}\left(d^{*}<d^{\prime}\right)$. Define the function $\lambda(r)$ in the following way

$$
\begin{aligned}
& \lambda(r)=\lambda z(r), \\
& z(r)=\left[\left(x_{d^{*}+1}\right)^{2}+\left(x_{d^{*}+2}\right)^{2}+\ldots+\left(x_{d^{\prime}}\right)^{2}\right]^{-\alpha / 2},
\end{aligned}
$$

where $x_{1}, \ldots, x_{d}$ are the axes of a Cartesian coordinate system. The coordinate axes $x_{1}, \ldots, x_{d}$ are chosen in such a way that axes $x_{1}, \ldots, x_{d^{\prime}}$ span the subspace of the perturbation in which the defect subspace with axes $x_{1}, \ldots, x_{d^{*}}$ is embedded. The integration in the first term of (13) is carried out in a d-dimensional space, while the second term involves a d'-dimensional subspace integration.

The results of our analysis are the following [6]. In the case

$$
d_{e f f}=\Delta_{\omega},
$$

where

$$
d_{e f f}=\max \left(d^{\prime}-\alpha, d *\right)
$$

we can calculate the change in the critical exponents to linear order in $\lambda$,

$$
\begin{gathered}
\Delta_{\varphi}=\Delta_{\varphi}^{0}+\lambda a_{1} S_{d^{\prime} d *}(\alpha)^{\prime}, \\
\Delta_{\omega}=\Delta_{\omega}^{0}+\lambda b_{1} S_{d^{\prime} d *}(\alpha) .
\end{gathered}
$$

Thus in this case local critical exponents are continuous functions of the microscopic pertubation parameter $\lambda_{j}$. This indicates that there is a local violation of the universality hypothesis.

Such a situation can occur in the general case, and not only for the case of a special symmetry - a characteristic of a special symmetry - a characteristic of the defect models investigated earlier whose dimensionalities were integers.

Recent developments in this field of investigation can be found in Refs. [6-8].

This work was supported in part by Conselho Nacional de Desenvolvimento Científico e Tecnológico CNPq - Brazil, and by Fundação de Amparo à Pesquisa do Estado de São Paulo - FAPESP - Brazil.

\section{References}

[1] E. Ising, Zeit. Phys. 31, 253 (1925).

[2] L. Onsager, Phys. Rev. 65, 117 (1944).

[3] R. Baxter, Phys. Rev. Lett. 26, 832 (19710.

[4] R. Z. Bariev, JETP 77, 1217 (1979).

[5] R. Z. Bariev, JETP 94, 374 (1988).

[6] B. N. Shalaev, Phys. Rept. 237, 129 (1994).

[7] F. Igloi, I. Peshel and L. Turban Adv. Phys. 42, 683(1993)

[8] M. Pleimling and W. Selke, Phys. Rev 59, 65 (1999). 